\title{
Emerging Pathogen: Shewanella Algae causing Burn Wound Infection - Report of Two Cases from a Tertiary Care Center
}

\author{
Pratibha Shamanna*, Jayashree Ravindran, Jacinta Lalremruati and \\ Muralidaran Sethumadavan
}

Department of Microbiology, St John's Medical College, Bangalore, Karnataka, India

*Corresponding author

A B S T R A C T

\section{Keywords \\ Emerging pathogen, Shewanella algae \\ Article Info \\ Accepted: \\ 04 April 2018 \\ Available Online: \\ 10 May 2018}

\section{Introduction}

Shewanella species are found throughout the world in marine environments, and most reported human infections occur in countries in warm climates (Holt et al., 2005). Shewanella species have been implicated in skin and soft tissue infections, bacteremia, biliary tract infections, thoracic empyema, endocarditis, dacrocystitis, intracranial abscess, arthritis, peritonitis, ventilator associated pneumonia, and ear infections (Tsai et al., 2008; Tan et al., 2008) Shewanella species is a saprophytic gram negative rod, belonging to family Vibrionaceae. It is frequently isolated from nonhuman sources and is rarely considered pathogenic in humans. Shewanella species are widely distributed in nature, with soil and water being their natural habitat. Initially they were considered to be colonisers or saprophytes thriving on previously damaged tissue (Winn et al., 2006).

Shewanella algae and Shewanella putrefaciens are the two important species of Shewanella that are most frequently implicated in human infections, although more than $80 \%$ are caused by $S$. algae (Tsai et al., 2008). S. algae was introduced as a new species in 1990 and has been most frequently associated with infections of skin and soft tissues resulting from breaches in dermis, such as ulcers or following trauma (Tsai et al., 2008; Simidu et al., 1990). Although S. algae has been reported to be the etiological agent of distinct 
human infections it is difficult to give clinical significance to the isolation of this microorganism as it may be present as a coloniser or a component of mixed bacterial flora (Renu Goyal et al., 2011). Here we present two case series in burns patients who were infected by $S$. algae during admission to burns ward at the same period of time. In the present study $S$. algae was repeatedly isolated from same patients and the patients responded to the targeted treatment supporting that $S$. algae was the true pathogen.

\section{Materials and Methods}

Wound swabs taken from raw burnt area sent to microbiology lab, were cultured on5\% Sheep Blood agar, MacConkey agar and liquid Thioglycollate medium. Simultaneously Gram's stain of the smear was made from wound swab to look for the presence of bacteria and tissue cells. The culture plates and thioglycollate broth after inoculation were incubated at $37^{\circ} \mathrm{C}$ for $18-24$ hours.

On Blood agar grey lytic colonies with red tan pigment was noticed after 24 hours of incubation and on MacConkey agar, nonlactose fermenting colonies were seen. Thioglycollate broth showed uniform turbidity. Bacterial colony from BA and MAwere tested for oxidase production by strips impregnated with $1 \%$ tetra methyl paraphenylenediamine dihydrochloride. The colonies were oxidase positive.Oxidase positive colonies were further identified by grain stain, motility on mannitol motility (MM) medium. Urease on Christensen's agar, Citrate utilization by Cimmon's citrate agar, $\mathrm{H} 2 \mathrm{~S}$ production and differentiation between fermenter and non-fermenter on Triple sugar iron agar slant (TSI) and for Indole production. The organism was identified as Shewanella species since this is the only nonfermenter which produces $\mathrm{H} 2 \mathrm{~S}$ gas in TSI agar and is also oxidase positive (Winn et al., 2006). Shewanella species were further identified as $S$. algae based on Nitrate reduction, metabolism of maltose, mannitol, sucrose, arabinose, ribose, decarboxylation of Amino acids, arginine, lysine and ornithine, hemolysis in 5\% sheep bloodagar, growth on Salmonella-Shigella agar(SS), growth at $42^{\circ} \mathrm{C}$, growth in presence of $6.5 \% \mathrm{NaCl}$, gelatin hydrolysis, DNAase production. Antibiotic sensitivity testing was done using Kirby-bauer disc diffusion method following CLSI guidelines with Ampicillin, Amikacin, Gentamicin, Netilmicin, Cephalexin, Cefotaxime, Ceftazidime, Ciprofloxacin, Cefoperazone + Sulbactam, Piperacillin + Tazobactam, Meropenem, Piperacillin, Imipenem, Ertapenem and zone of inhibition was measured and reported as sensitive or resistant (NCCLS, 2003). In both of cases the isolates were identified as Shewanella algae based on the ability to produce mucoid colonies with Beta-hemolysis on 5\% sheep blood agar, growth at $42^{\circ} \mathrm{C}$ and in $6 \% \mathrm{NaCl}$, inabililty to produce acid from maltose and ability to reduce nitrite (Rajshree Patel et al., 2012).

\section{Case-1}

22yr old male patient, cook by occupation with history of accidental thermal burns sustained 3 months back at his residence was admitted to Victoria hospital and later referred to St John's Hospital for further treatment and follow up, came with raw area involving face, neck, chest.

On local examination: Post burns raw area with slough, granulation tissue along with discharge was noticed involving mainly anterior chest, upper abdomen, right posterior chest, scalp area and bilateral medial aspect of forearm. There was restriction in lateral shoulder abduction and neck movements. Also grade III contracture of ear was observed.

Investigations revealed $\mathrm{Hb}-10.6 \%$, PCV33.20, S. Creatinine 0.1, B. Urea 181, 
$\mathrm{Na} / \mathrm{K} / \mathrm{CL}-35 / 4.6 / 100$, and HIV and HBsAg status - Non reactive.

Wound swab taken from discharge site for culture and sensitivity in Gram's stain-showed numerous pus cells, numerous gram positive cocci in singles, pairs, clusters, numerous gram negative bacilli. Wound swab culture showed growth of Shewanella algae and Staphylococcus aureus.

Shewanella algae were sensitive to Pipercillin + Tazobactam, Piperacillin, Cefoperazone + Salbactam, Gentamycin, Meropenem, intermediate to Amikacin. Staphylococcus aureus was sensitive to Netilmicin, Amikacin, Tetracycline, Chloramphenicol, Erythromycin, Vancomycin, Teicoplanin. Repeat sample sent also grew Shewanella algae with same sensitivity pattern. A provisional diagnosis $22 \%$ burnswith $S$. algae infection was made. The patient underwent Split skin grafting (SSG) in anterior chest and bilateral arm and SSG on the back. Post operatively graft was healthy and patient advised for discharge and regular follow up.

\section{Case-2}

An 8yr old female child came with alleged history of flame burns, child clothes caught fire while playing near campfire.

On local examination: $2^{\circ}$ deep to $3^{\circ}$ burns over the right thigh anterior aspect, left anterior thigh, lateral aspect and left lower anterior trunk.

Investigations revealed: HB-9.7\%, S.Creat0.6\%, Na+ 128, K-3.6, CL-96, HIV, HBsAgNon -reactive. Wound swab taken from discharge site on Gram's stain-showed scanty pus cells, scanty gram negative bacilli. Wound swab culture grew Shewanella algae and Klebsiella species. Shewanella algae was sensitive to Pipercillin + Tazobactam, Piperacillin, Cefoperazone + Salbactum,
Meropenem and Klebsiella species was resistant to all gram negative drugs. A provisional diagnosis of $15 \%$ thermal burns with infection was made. Patient was taken for $6 \%$ epifacial excision of left thigh $9 \%$ total excision over abdomen with homograft and right thigh with autograft done. After a week homograft over left thigh was replaced with autograft. Post operatively graft was taken up well and child was advised discharge with regular follow up.

\section{Results and Discussion}

Although pseudomonas aeruginosa is the most common oxidase positive non fermenter isolated from clinical specimens, Shewanella species has recently attracted attention of clinical microbiologists. Recognition of this rare organism has been hampered as the isolation of Shewanella species from clinical samples has long been considered merely colonization rather than active infective agent (Mukhopadhyay et al., 2007). The organism generally resides in marine and aquatic environment so the route of transmission in these cases in not clear. There have been only four reports of isolation of Shewanella from India and these were from patients with infective endocarditis, peritonitis, chronic obstructive pulmonary disease respectively (Mukhopadhyay et al., 2007; Dhawan et al., 1998). Shewanella algae and Shewanella putrefaciens are the two species of Shewanella that are most frequently implicated in human infections. Among Shewanella species, S. algae causes $80 \%$ human infections (Khashe and Micheal, 1998).

Important differential characteristics between the two species include the ability of $S$. algae to produce mucoid colonies with $\beta$ - heamolysis on 5\% sheep blood agar, to grow at $42^{\circ} \mathrm{c}$ and in $6 \% \mathrm{NaCl}$ and to reduce nitrate, and an inability to produce acid from maltose all of which are in contrast to the characteristics of Shewanella putrefaciens (Fig. 1-4). 
Fig.1 Blood agar showing lytic colonies with red tan pigment

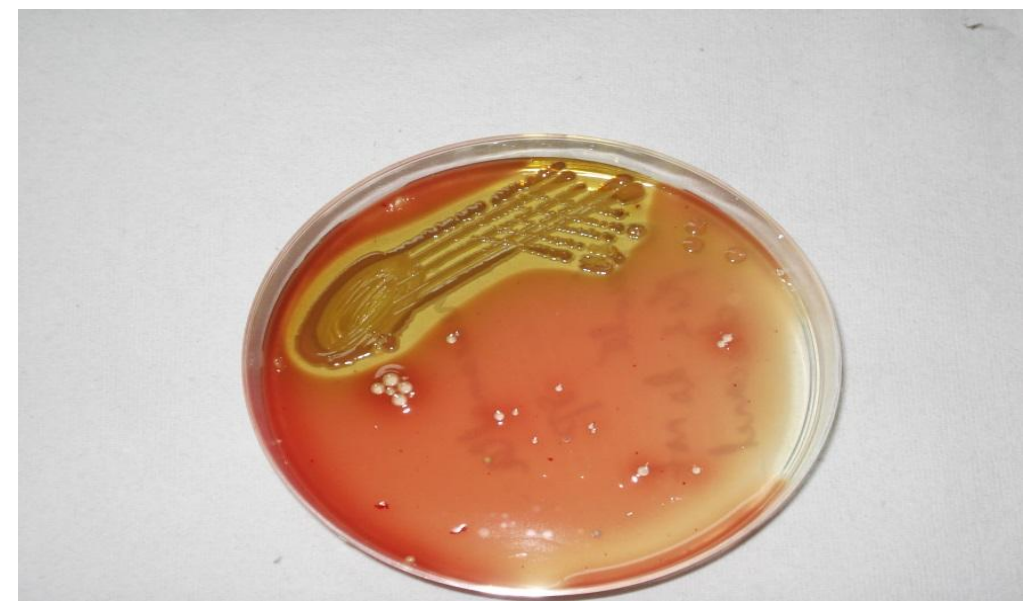

Fig.2 MacConkey's agar showing non-lactose fermenting colonies

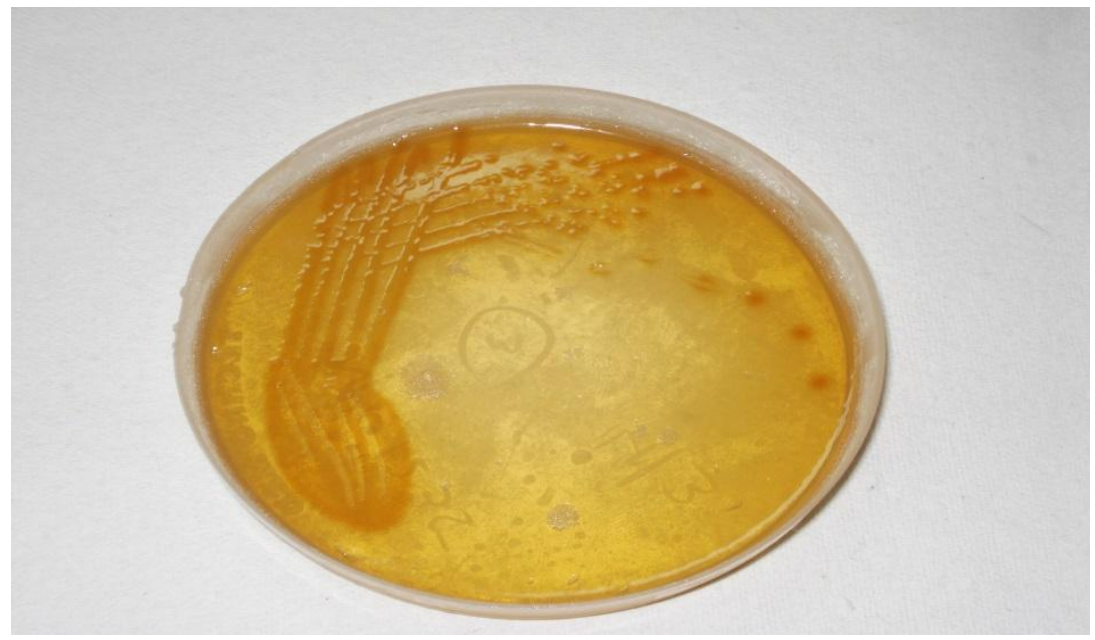

Fig.3 Biochemical properties of Shewannella algae. a) Showing OF (oxidative fermentative media with $1 \%$ glucose), MM (mannitol motility media), TSI (triple sugar iron media), Indole, Citrate, Urease. b) Ornithine decarboxylase test with control

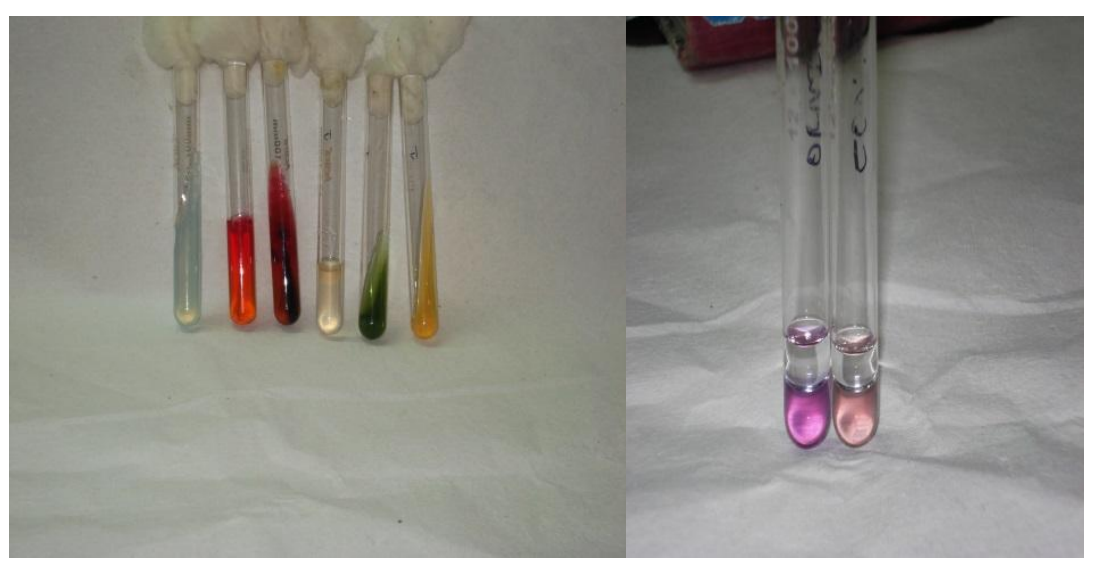


Fig.4 DNase test with control

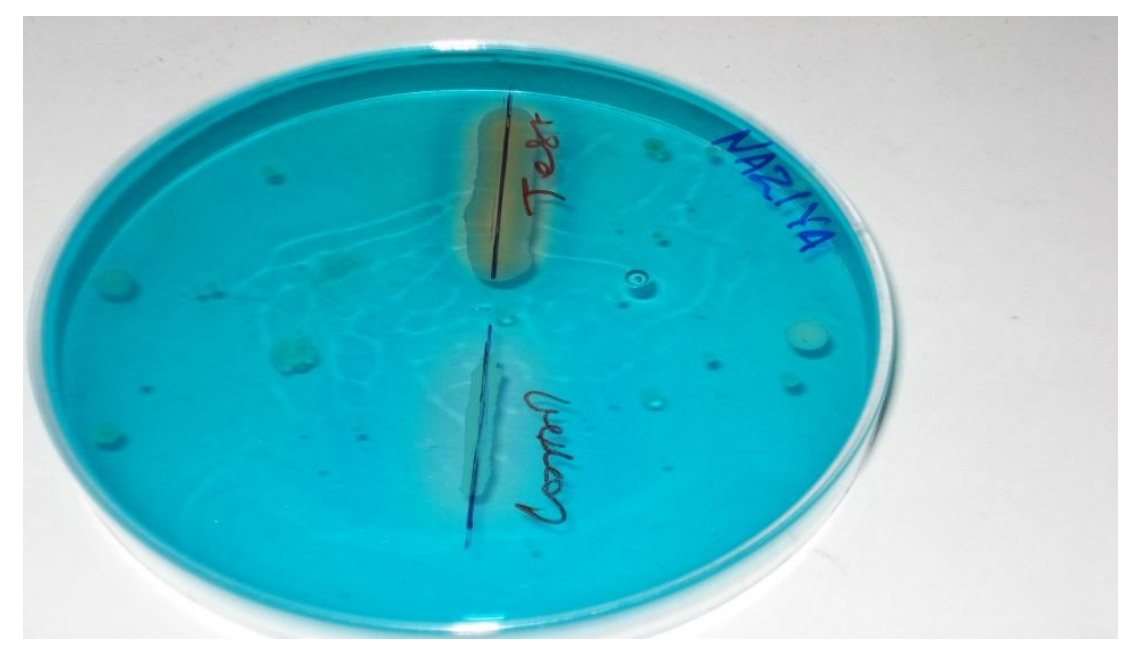

Automated identification systems fail to differentiate between Shewanella agae and Shewanella putrefaciens, as $S$. algae is not in included in the databases of these systems. Presumably for this reason most Shewanella infections reported during recent years have been attributed to Shewanella putrefaciens.

However in studies where extensive phenotypic characterisation is performed most human infections are caused by Shewanella algae. As the two species have different pathogenic potential for humans, correct identification is important, and this is possible only in routine clinical microbiology laboratories (Holt et al., 2003).

In present study also Shewanella algae was isolated in both the burn wound cases, as most common infection due to Shewanella species are infections of skin and soft tissue and are usually associated with breaches in the skin such as ulcers or following trauma like in other studies.

There are no standard guidelines for treatment of Shewanella infection. In both of our cases the organism was sensitive to Piperacillin, Piperacillin + Tazobactam, Meropenem and Gentamicin, intermediate sensitive to Amikacin and resistant to other gram negative antibiotics. Previously published studies reported that
Shewanella algae are susceptible to aminoglycosides, carbapenems, erythromycin, and quinolones with variable susceptibility to penicillin and cephalosporin. However rapid development of resistant to Imipenem and Piperacillin + Tazobactam has been reported (Ostwal et al., 2015).

Though burn wound infections due to Shewanella algae have been reported from several countries, such reports from India are rare. The main reason for this is that in most of the hospitals, all gram negative and oxidase positive organisms are reported as Pseudomonas species and no further identification is done.

To conclude the isolation of Shewanella algae from above two cases strengthens the assumption that the organism should be regarded as a potential emerging pathogen. Utmost microbiological vigilance to diagnose the unusual pathogen will facilitate the administration of appropriate treatment at the earliest thereby getting satisfactory clinical response and prevention of further complications.

\section{Acknowledgement}

We acknowledge the sincere effort of our MSc postgraduate student Jacinta Lalremruati, for 
her help in laboratory diagnosis. Also we acknowledge Department of Plastic Surgery for the clinical samples and necessary clinical data.

\section{References}

Dhawan B, Chaudry R, Mishra BM and Agarwal R.1998 Isolation of Shewanella putrefaciens from a Rheumatic Heart Disease Patient with Infective Endocarditis. J Clin Microbial. 36: 23946.

Holt HM, Hansen BG, Brunn B. 2005. Shewanella algae and Shewanella putrefaciens: clinical and microbiological characteristics. Clin Microbial Infect. 11: 347-352.

Holt HM, Sogaarrd P, Gahrn-Hansen B. Bruun. 2003. Shewanella alga and Shewanella putrefaciens: clinical and microbiological characteristics ClinMicrobiol Infect. 11: 347-352.

Khashe S and Micheal JJ. 1998. Biochemical and Pathogenic Properties of Shewanella alga and Shewanella putrefaciens JClinMicrobiol. 36(3): 783-787.

Mukhopadhyay C, Chawla K, Sharma Y, Bairy I. 2007 First Report of Shewanella alga as Emerging Infection in India: Two Cases. Journal of Clinical and Diagnostic Research. Aug; 1(4): 293-295.

NCCLS, Performance Standards for Antimicrobial Disc Susceptibility Tests. Approved Standard. $8^{\text {th }}$ ed NCCLS Document M2-A8 Wayne, Pa: NCCLS: 2003.
Ostwal K, Shah P, Pathak S, Jadhav A, Shaikh $\mathrm{N}, 2015$. New enterant in the class of uropathogens- Shewanella algae, Asian Journal of Medical Sciences. Jul-Aug 6(4): 92-95.

Rajshree Patel, Albin Abraham, Johnson Thomas, Wanqing Zhi, Shadab Ahmed, Janice Verley. A Rare case of pneumonia caused by Shewanella putrefaciens. Hindawi Publishing Corporation Case Reports in Medicine. Volume 2012, Article ID 597301, 3 pages doi:10.1155/2012/597301.

Renu Goyal, Narinder Kaur, Rajeev Thakur. 2011. Human soft tissue infection by the emerging pathogen Shewanella algae, J infect DevCtries. 5(4): 310-312.

Simidu U, Ysukamoto K, Yasumoto T, Yotsu M. 1990. Taxonomy of four marine bacterial strains that produce tetradoxin. Int J SystBacteriol. 40: 331-336.

Tan CK, Lai CC, Kuar WK, Hsueh PR. 2008. Purulent pericarditis with greenish pericardial effusion caused by Shewanella algae. J Clin Microbiol.46: 2817-2819.

Tsai MS, You HL, Tang YF, Liu JW. Shewanella soft tissue infection: case report and literature review. International J Infect Dis 2008; 12: 119-124.

Winn W Jr, Allen S, Janda W, Koneman E, Procop G, Schrenberger P, Woods G. 2006. Koneman's colour Atlas and textbook of Diagnostic microbiology. $6^{\text {th }}$ ed. Lippincott Williams and Wilkins (USA); Pp. 303-391.

\section{How to cite this article:}

Pratibha Shamanna, Jayashree Ravindran, Jacinta Lalremruati and Muralidaran Sethumadavan. 2018. Emerging Pathogen: Shewanella Algae causing Burn Wound Infection - Report of Two Cases from a Tertiary Care Center. Int.J.Curr.Microbiol.App.Sci. 7(05): 48-53. doi: https://doi.org/10.20546/ijcmas.2018.705.007 